\title{
PRODUCCIÓN Y CALIDAD DE LA MORERA (Morus alba) COSECHADA EN DIFERENTES MODALIDADES DE PODA ${ }^{1}$
}

\author{
Carlos Boschini ${ }^{2}$
}

\begin{abstract}
RESUMEN
Producción y calidad de la biomasa de morera (Morus alba) cosechada en diferentes modalidades de poda. Una plantación establecida en 1993, con un distanciamiento entre plantas de 60,90 y $120 \mathrm{~cm}$ entre hileras y plantas, fue cosechada 26 veces a diferentes intervalos (56 a 112 días) de poda durante cinco años consecutivos, a la altura de $60 \mathrm{~cm}$ sobre el nivel del suelo. Se practicaron tres tipos de poda: testigo, semilimpio y limpio. Con un intervalo de 84 días de crecimiento se cosecharon los rebrotes y se determinó el contenido de materia seca, proteína cruda, fibra neutro detergente y cenizas totales. La poda semilimpia provocó una pérdida del $10 \%$ en la producción de materia seca de hoja por hectárea y un $13 \%$ en el rendimiento de tallo con respecto al tratamiento testigo. La cosecha conjunta de hojas y tallos decreció un $11 \%$. La poda limpia provocó una disminución de $32 \%$ en el rendimiento de materia seca de hoja por hectárea y $36 \%$ en la producción de tallo con relación al tratamiento testigo. La producción compuesta decreció 33\%. La calidad nutricional de los rebrotes, hojas y tallos, fue similar entre los diferentes tratamientos de poda.
\end{abstract}

\begin{abstract}
Production and quality of mulberry (Morus alba) harvested with different pruning techniques. A plantation established in 1993 with $60 \mathrm{~cm}, 90 \mathrm{~cm}$ and $120 \mathrm{~cm}$ between rows, was harvested $60 \mathrm{~cm}$ above the ground, 26 times at different pruning intervals between 56 and 112 days. Three pruning techniques were used: control, semi-clean and completely clean. Every $84^{\text {th }}$ day of growth, the shoots were harvested and the total content of dry material, crude protein, neutral detergent fiber and ash were analyzed. The semiclean pruning caused a $10 \%$ loss in the production of dry leaf material per hectare and a $13 \%$ in the stem yield, compared to the control. The joint harvest of leaves and stems decreased $11 \%$. The completely clean pruning caused a $32 \%$ decline in the yield of dry material in the leaves per hectare and $36 \%$ in stem production, compared to the control. The total yield decreased $33 \%$. The nutritional quality of the shoots, leaves and stems was similar among the different pruning techniques.
\end{abstract}

\section{INTRODUCCIÓN}

La investigación de la morera para uso en sericultura se ha llevado a cabo tradicionalmente en China y en la India. Con el mismo propósito, países europeos como Francia, Italia, Bulgaria y Polonia efectuaron trabajos de investigación desde inicios del siglo XIX hasta mediados del siglo XX, cuando la producción de seda prácticamente desapareció. Estudios recientes sobre morera para alimentación animal se han realizado en Japón, India, Tanzania, Kenya, Costa Rica, Colombia, Cuba, México, Guatemala y Brasil e incluyen aspectos agronómicos, modalidades de cosecha, conservación de forrajes y pruebas con animales (Sánchez 2000). En países con alta disponibilidad de mano de obra y en pequeña escala familiar, la cosecha del follaje se efectúa por recolección manual directa (Datta 2000), seleccionando las hojas en el mejor estado nutricional y dejando los tallos intactos en pie con ocho a 10 hojas jóvenes en el ápice. A través del año se practican podas de conformación según lo requiera la planta, por lo general arriba de un metro sobre el terreno. Una vez al año, en la estación de invierno, cuando las yemas están en estado de dormancia, se realiza la poda total en dos modalidades: uno en corte de raíz que se efectúa a tres centímetros sobre el nivel del suelo y otro en corte se-

1 Recibido para publicación el 28 de marzo del 2001. Proyecto No. 737-97-006, inscrito en la Vicerrectoría de Investigación, Universidad de Costa Rica.

2 Estación Experimental Alfredo Volio Mata. Facultad de Agronomía. Universidad de Costa Rica. E-mail: boschini@cariari.ucr.ac.cr. Tel: (506) 279-5840, 279-5850. 
mi-bajo el cual es hecho a un punto entre 10 y $40 \mathrm{~cm}$ sobre el terreno (Ting-zing et al. 1988). En América Latina, la biomasa de morera empleada en la alimentación animal se cosecha por poda total, en intervalos de 45 a 120 días de rebrote (Rodríguez et al. 1994, Benavides et al. 1986), con alturas de corte entre 30 y 100 cm (Blanco 1992, Benavides et al. 1986), formándose un fuste de tres a cuatro ejes centrales y en cada uno de ellos, se forman decenas de ramificaciones secundarias producto de los rebrotes sucesivos con una apariencia de corona ramiforme. Esta corona en podas y rebrotes siguientes amplia su diámetro, hasta cerrar el espacio entre los surcos, a la altura de corte establecido (40 - 50 $\mathrm{cm}$ sobre el terreno) en las plantaciones con alta densidad de siembra (más de 20000 plantas/ha) después de cinco a seis años de producción continua. La eliminación de esta corona es necesaria para el manejo de la plantación en los años siguientes, mediante una poda profunda. Este experimento se llevó a cabo con el propósito de conocer los efectos de tres modalidades de poda sobre el comportamiento de la producción de biomasa en el rebrote siguiente.

\section{MATERIALES Y MÉTODOS}

El experimento se llevó a cabo en la Estación Experimental de Ganado Lechero "Alfredo Volio Mata" (EEAVM) de la Universidad de Costa Rica, ubicada a $1542 \mathrm{msnm}$ con una precipitación anual media de $2050 \mathrm{~mm}$; distribuida de mayo a noviembre. El restante período es seco. La temperatura media es de $19,5^{\circ} \mathrm{C}$. La humedad relativa media es de $84 \%$. El suelo es de origen volcánico, clasificado como Typic Distrandepts (Vásquez 1982) y caracterizado por una profundidad media, buen drenaje natural y fertilidad media $(7,7$ $\mathrm{cmol} / \mathrm{l}$ de calcio, 3,0 de magnesio y $1,54 \mathrm{cmol} / 1$ de potasio, $10,0 \mathrm{mg} / \mathrm{l}$ de fósforo, 28,8 de cobre, 234 de hierro, 6,3 de manganeso y $2,6 \mathrm{mg} / \mathrm{l}$ de zinc. Un $\mathrm{pH}$ de 5,9 . Ecológicamente, la zona se tipifica como bosque húmedo montano bajo (Tosi 1970, citado por Vásquez 1982).

Una plantación de $5000 \mathrm{~m}^{2}$ establecida en 1993, con estacas jóvenes de uno a dos cm de diámetro, 40 $\mathrm{cm}$ de largo y con al menos tres yemas. Las estacas fueron sembradas entre cinco y ocho $\mathrm{cm}$ de profundidad en tres lotes, cada uno con un distanciamiento entre plantas de 60, 90 y $120 \mathrm{~cm}$ entre hileras y plantas, equivalente a densidades de siembra de 27777,12345 y 6944 plantas/ha respectivamente. Los tres lotes se desarrollaron durante un año y posteriormente se realizaron 26 cosechas de biomasa a diferentes intervalos (56 a 112 días) de poda durante cinco años consecuti- vos a una altura de $60 \mathrm{~cm}$ sobre el nivel del suelo. Cuando los rebrotes alcanzaron de tres a cinco $\mathrm{cm}$ de largo (dos semanas post corte), se fertilizaron con nitrato de amonio en una dosis equivalente de $300 \mathrm{~kg}$ N/ha/año (IFA 1992, Rodríguez et al. 1994).

En el trabajo de campo los tratamientos se arreglaron en forma factorial $3 \times 3$ con cuatro repeticiones. El factor distancias de siembra tuvo tres niveles: $60,90 \mathrm{y}$ $120 \mathrm{~cm}$ entre surcos y entre plantas y el factor tipos de poda con los niveles: testigo, semilimpio y limpio. En el tratamiento testigo, se mantuvo la estructura presente de la planta, uniformando el corte a la altura de $60 \mathrm{~cm}$ como se venía cortando anteriormente (sin poda adicional), el tratamiento semilimpio consistió en una poda uniforme a $40 \mathrm{~cm}$ sobre el nivel de suelo, manteniendo todas las ramas secundarias por debajo de ese nivel con sus respectivas hojas. El tratamiento limpio fue una poda uniforme a $40 \mathrm{~cm}$ de altura sobre el terreno, limpiando todas las ramas secundarias por debajo de ese nivel, quedando desnudo los tallos principales (troncos) de cualquier brote, ramas u hojas. En la Figura 1 se observa los tipos de poda empleado. Se midió la producción de hojas y tallos producidos en los dos rebrotes siguientes, con un intervalo de 84 días de crecimiento. De cada tratamiento se extrajo una muestra por repetición para la determinación del contenido de materia seca, proteína cruda, fibra neutro detergente y cenizas totales. Las muestras de hojas y tallos fueron secados en un horno a $60^{\circ} \mathrm{C}$ durante 48 horas, hasta alcanzar un peso constante. Posteriormente se molieron y se determinó el contenido de materia seca en una estufa a $105^{\circ} \mathrm{C}$. La proteína cruda se determinó por el método Kjeldall y la cenizas totales por incineración (AOAC 1980). La fibra neutro detergente (FND) fue analizada con el método descrito por Goering y Van Soest (1970). Los datos se analizaron con el PROC GLM del paquete estadístico SAS (1985).

\section{RESULTADOS Y DISCUSIÓN}

La producción anual de materia seca en los rebrotes, tallos y hojas por separado, obtenida en cada una de las distancias de siembra se observan en la Figura 2. Este efecto independiente fue altamente significativo $(\mathrm{P}$ $\leq 0,01)$. La biomasa foliar resultó superior a la producción de tallo en todas las densidades. Las distancias de siembra incidieron fuertemente en la producción de las plantas, mostrando una tendencia no lineal a decrecer el rendimiento conforme aumentó la distancia de siembra, similar al observado por Boschini (1998) que reportó un decremento de $37 \%$ al aumentar la distancia de 60 a $90 \mathrm{~cm}$ y de $13 \%$ al variar de 90 a $120 \mathrm{~cm}$ entre plantas. Similares efectos fueron observados en la producción 


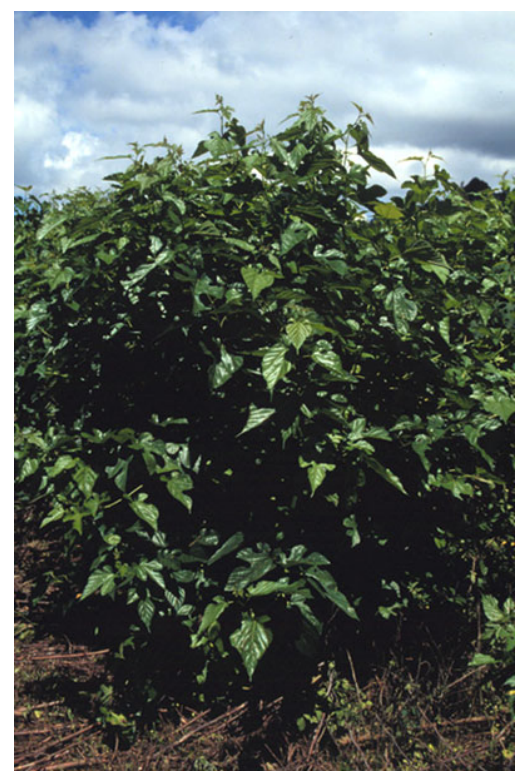

Antes de la poda

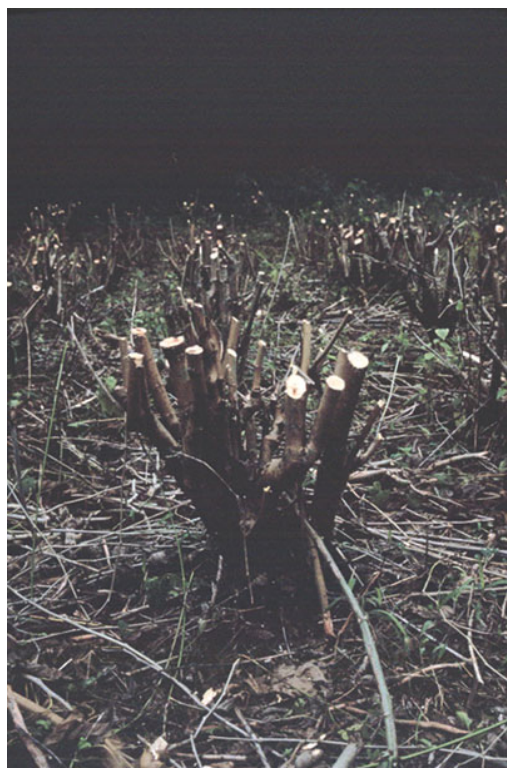

Poda semilimpia

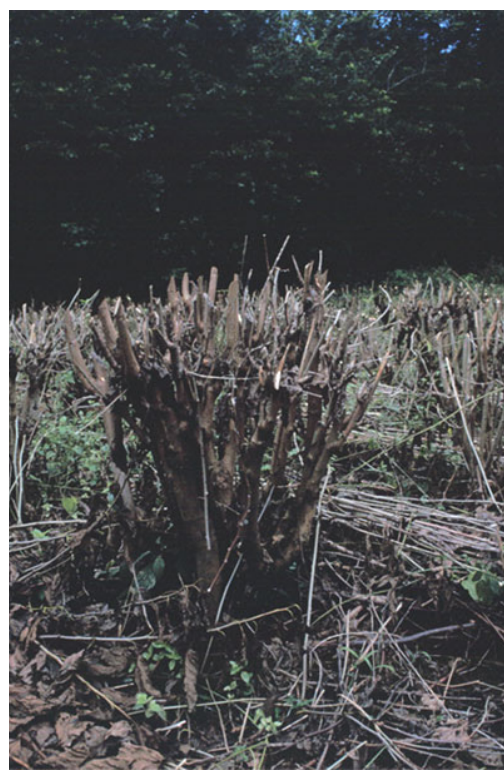

Poda testigo

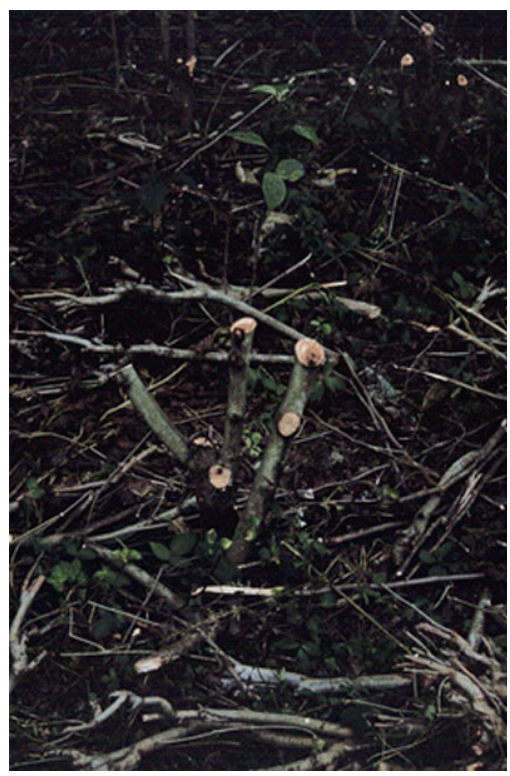

Poda limpia

Figura 1. Tipos de poda practicados en el experimento. EEAVM, El Alto de Ochomogo, Costa Rica.

de hojas y de tallos, mostrándose en estos últimos una declinación mayor. Lin et al. (1994) indican que conforme aumenta la densidad de siembra, la productividad por planta disminuye sensiblemente y de manera no lineal con cuatro cosechas por año. El mayor espaciamiento entre plantas disminuye la competencia por luz (González 1951) y las plantaciones con alta densidad de siembra responden favorablemente a la competencia.
La producción de materia seca anual medida en la planta entera y en hojas y tallos por separado, fue afectada por cada uno de los tipos de poda practicados y los resultados se presentan en la Figura 3. Este efecto en forma independiente resultó altamente significativo $(\mathrm{P}$ $\leq 0,01)$. La poda semilimpia presentó una producción de $90,07 \%$ y la poda limpia de $67,61 \%$ con relación a la producción de hojas en el tratamiento testigo. La poda limpia tuvo una producción proporcional de 75,06\% 


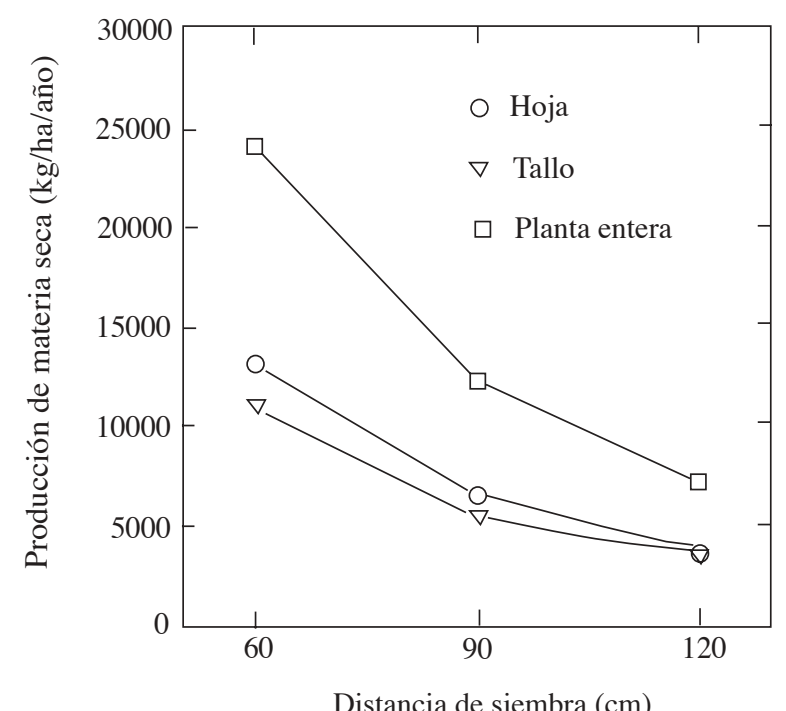

Figura 2. Producción de materia seca en tres distancias de siembra. EEAVM, El Alto de Ochomogo, Costa Rica.

de la semilimpia. En tallos, la producción fue de $87,14 \%$ en el tratamiento semilimpio y de $63,94 \%$ en el tratamiento limpio respecto al tratamiento testigo. El tratamiento limpio presentó una producción de 73,38\% en proporción a la observada en el semilimpio. La combinación de las dos fracciones del rebrote, muestra que la cosecha en forma de poda semilimpia representó un $88,70 \%$ del testigo y el corte limpio un $66,81 \%$. El corte limpio tuvo una producción $75,33 \%$ con relación al tratamiento semilimpio.

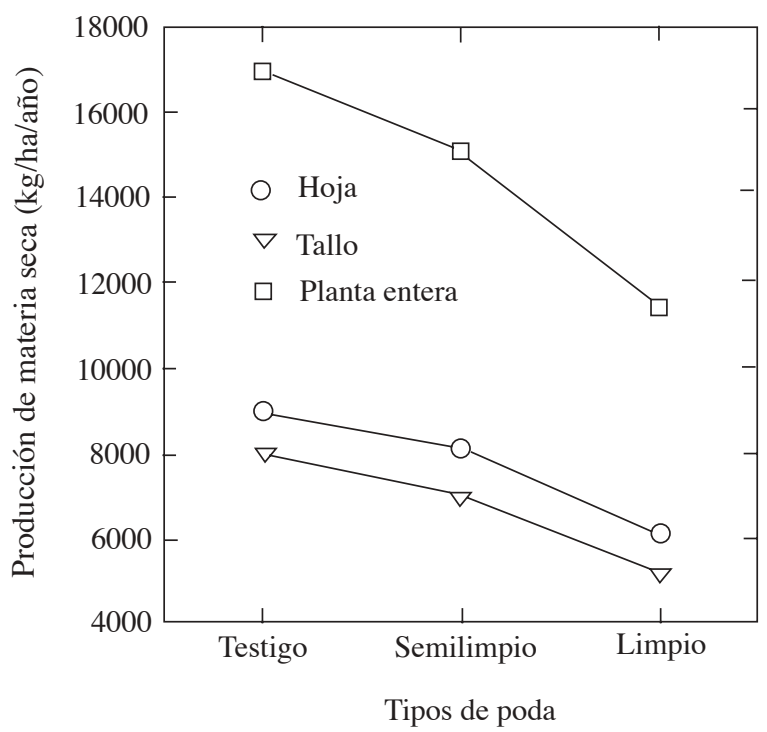

Figura 3. Producción de materia seca en tres tipos de poda. EEAVM, El Alto de Ochomogo, Costa Rica.
El contenido nutricional de la biomasa en la planta entera y sus fracciones, al variar las distancias de siembra, se observa en el Cuadro 1. La concentración de materia seca en hojas fue significativa $(\mathrm{P}<0,01)$ entre distancias con un rango de 17,56 a $18,61 \%$. Esta variación de $1 \%$ es poco importante para favorecer o discriminar una u otra distancias de siembra. En tallos la proporción de materia seca fue estadísticamente similar $(\mathrm{P}>0,05)$ y en la planta entera se mostró una ligera variación $(\mathrm{P}<0,05)$. El contenido proteico en la materia seca mostró poca variación $(\mathrm{P}>0,05)$ tanto en la fracción de hojas como en los tallos al aumentar las distancias de siembra. La proteína no varía con la distancia de siembra, corroborado por Boschini (2000). Similar respuesta $(\mathrm{P}>0,05)$ se encontró en la proporción de fibra neutro detergente analizada en hojas y en la planta entera al cambiar las distancias de siembra. En el tallo la proporción de pared celular varió ligeramente $(\mathrm{P}<0,05)$ en un rango de 70 a $74 \%$, biológicamente poco importante. Las cenizas mostraron idéntica respuesta $(\mathrm{P}<0,05)$ a la observada en el contenido de proteína cruda.

Cuadro 1. Contenido de proteína cruda, fibra neutro detergente y cenizas en la materia seca de la morera cosechada a 84 días de edad, según la distancia de siembra. EEAVM, El Alto de Ochomogo, Costa Rica.

\begin{tabular}{clcccr}
\hline $\begin{array}{c}\text { Distancia } \\
\text { de siembra } \\
(\mathbf{c m})\end{array}$ & $\begin{array}{l}\text { Parte } \\
\text { de la } \\
\text { planta }\end{array}$ & $\begin{array}{c}\text { Materia } \\
\text { seca } \\
(\boldsymbol{\%})\end{array}$ & $\begin{array}{c}\text { Proteína } \\
\text { cruda } \\
(\boldsymbol{\%})\end{array}$ & $\begin{array}{c}\text { FND } \\
(\boldsymbol{\%})\end{array}$ & $\begin{array}{c}\text { Cenizas } \\
(\boldsymbol{\%})\end{array}$ \\
\hline \multirow{2}{*}{60} & Hoja & 18,61 & 23,95 & 36,03 & 18,11 \\
& Tallo & 16,85 & 8,28 & 70,07 & 7,76 \\
& Entera & 17,76 & 16,75 & 53,55 & 13,32 \\
90 & Hoja & 17,56 & 24,32 & 38,62 & 18,87 \\
& Tallo & 16,14 & 8,64 & 73,84 & 7,68 \\
& Entera & 16,87 & 16,97 & 55,23 & 13,60 \\
\multirow{2}{*}{120} & Hoja & 17,80 & 23,39 & 36,67 & 18,05 \\
& Tallo & 16,88 & 7,97 & 72,03 & 8,14 \\
& Entera & 17,35 & 15,76 & 54,03 & 13,17 \\
\hline
\end{tabular}

En el Cuadro 2 se presenta los contenidos nutricionales de los rebrotes en los distintos tratamientos de poda. En la planta entera y en la fracción de tallos las variaciones en los contenidos de materia seca fueron poco importantes $(\mathrm{P}>0,05)$ entre tratamientos. En la hoja se mostraron diferencias estadísticas $(\mathrm{P}<0,01)$ en un rango de 17 a $18,5 \%$ con los distintos tipo de poda. Un $1,5 \%$ de variación en el contenido de materia seca no es suficiente para destacar los beneficios de uno $\mathrm{u}$ otro tipo de poda. El contenido de proteína cruda presentó pequeñas variaciones $(\mathrm{P}<0,05)$ entre los tratamientos de poda tanto en hojas como en tallo. La misma respuesta en hojas fue observada por Yongkang Huo 
Cuadro 2. Contenido de proteína cruda, fibra neutro detergente y cenizas en la materia seca de la morera cosechada a 84 días de edad, según el tipo de poda. EEAVM, El Alto de Ochomogo, Costa Rica.

\begin{tabular}{llcccr}
\hline $\begin{array}{c}\text { Tipo de } \\
\text { poda }\end{array}$ & $\begin{array}{c}\text { Parte } \\
\text { de la } \\
\text { planta }\end{array}$ & $\begin{array}{c}\text { Materia } \\
\text { seca } \\
(\boldsymbol{\%})\end{array}$ & $\begin{array}{c}\text { Proteína } \\
\text { cruda } \\
(\boldsymbol{\%})\end{array}$ & $\begin{array}{c}\text { FND } \\
(\boldsymbol{\%})\end{array}$ & $\begin{array}{c}\text { Cenizas } \\
(\boldsymbol{\%})\end{array}$ \\
\hline Testigo & Hoja & 17,13 & 24,18 & 39,65 & 18,29 \\
& Tallo & 16,89 & 8,36 & 74,47 & 7,66 \\
& Entera & 17,04 & 16,54 & 56,41 & 13,19 \\
Semilimpia & Hoja & 18,51 & 22,75 & 35,37 & 18,46 \\
& Tallo & 16,89 & 7,52 & 73,11 & 7,75 \\
& Entera & 17,75 & 15,53 & 53,21 & 13,36 \\
Limpia & Hoja & 18,33 & 24,73 & 36,30 & 18,28 \\
& Tallo & 16,09 & 8,99 & 72,35 & 8,17 \\
& Entera & 17,19 & 17,41 & 53,19 & 13,54 \\
\hline
\end{tabular}

(2000). Similares resultados se hallaron en la concentración de fibra neutro detergente. El contenido de cenizas dentro de hojas y dentro de tallos no mostró variaciones $(\mathrm{P}>0,05)$ con respecto al tipo de poda practicado. En el Cuadro 3 se presentan las concentraciones de materia seca y proteína entre los tratamientos de poda por cada distancia de siembra. Esta interacción no presentó variaciones de importancia en ninguna de las partes componentes de la planta.

Las plantaciones de morera para la producción de forraje en rumiantes, evaluadas en condiciones tropicales de Costa Rica y Guatemala (Benavides et al. 1994, Rodríguez et al. 1994), son cultivos menores de 10 años de edad, relativamente nuevos para requerir renovaciones completas. La utilización de podas de conformación no es una práctica cultural establecida en nuestro medio, efectuándose únicamente la poda en el momento de la cosecha con el objetivo de recolectar la biomasa peso sin tomar en cuenta el futuro productivo de la plantación. La planta de morera permite este maltrato continuo corte tras corte y responde una cosecha tras otra sin disminuir la producción de biomasa, desarrollando obviamente la formación de una corona desordenada de rebrote que impide progresivamente el ingreso entre las hileras del cultivo. Los beneficios cualitativos de los tratamientos de poda practicados en el presente experimento no fueron evaluados. Los resultados mostraron que la poda semilimpia provocó una disminución del $10 \%$ en la producción de hoja y un $13 \%$ de tallo con respecto al tratamiento testigo. La fitomasa total cosechada decreció en forma global un $11 \%$. La poda limpia provocó una disminución de 32\% de hoja y 36\% de tallo con relación al tratamiento testigo. La producción global bajó 33\%. Desde el punto de vista fisiológico, las podas anuales disminuyen las reservas de carbohidratos solubles requeridas para el rebrote de las plantas (Taiz y Zeiger 1991) y en consecuencia disminuyen la capacidad de rebrote siguiente; sin embargo, Yamashita (1984), Yamashita y Fujino (1986), Suzuki y Kohno (1987) y Suzuki et al. (1988) advierten que el efecto de disminución de carbohidratos en la morera se magnifica según la estación del año y que en la recuperación de la planta influye la magnitud de daño ocasionado al tallo durante la cosecha.

Cuadro 3. Contenido de proteína cruda, fibra neutro detergente y cenizas en la materia seca de la morera cosechada a 84 días de edad, según la distancia de siembra y el tipo de poda. EEAVM, El Alto de Ochomogo, Costa Rica.

\begin{tabular}{|c|c|c|c|c|c|c|}
\hline $\begin{array}{c}\text { Distancia } \\
\text { de siembra } \\
\text { (cm) }\end{array}$ & $\begin{array}{l}\text { Tipo de } \\
\text { poda }\end{array}$ & $\begin{array}{c}\text { Parte } \\
\text { de la } \\
\text { planta }\end{array}$ & $\begin{array}{c}\text { Materia } \\
\text { seca } \\
(\%)\end{array}$ & $\begin{array}{c}\text { Proteína } \\
\text { cruda } \\
(\%)\end{array}$ & $\begin{array}{r}\text { FND } \\
(\%)\end{array}$ & $\begin{array}{c}\text { Cenizas } \\
(\%)\end{array}$ \\
\hline \multirow[t]{9}{*}{60} & \multirow[t]{3}{*}{ Testigo } & Hoja & 17,97 & 23,75 & 38,99 & 17,53 \\
\hline & & Tallo & 17,00 & 8,62 & 73,84 & 7,79 \\
\hline & & Entera & 17,51 & 16,76 & 55,08 & 13,03 \\
\hline & \multirow[t]{3}{*}{ Semilimpia } & Hoja & 19,18 & 23,00 & 33,93 & 18,62 \\
\hline & & Tallo & 16,51 & 8,21 & 72,62 & 8,04 \\
\hline & & Entera & 17,94 & 16,25 & 51,60 & 13,72 \\
\hline & \multirow[t]{3}{*}{ Limpia } & Hoja & 18,68 & 25,10 & 35,18 & 18,17 \\
\hline & & Tallo & 17,04 & 8,01 & 75,75 & 7,45 \\
\hline & & Entera & 17,83 & 17,23 & 53,97 & 13,21 \\
\hline \multirow[t]{9}{*}{90} & \multirow[t]{3}{*}{ Testigo } & Hoja & 16,54 & 25,17 & 40,11 & 19,93 \\
\hline & & Tallo & 15,43 & 8,42 & 74,95 & 7,51 \\
\hline & & Entera & 16,02 & 17,35 & 56,37 & 14,12 \\
\hline & \multirow[t]{3}{*}{ Semilimpia } & Hoja & 18,33 & 22,95 & 37,46 & 18,31 \\
\hline & & Tallo & 17,63 & 7,73 & 75,39 & 6,93 \\
\hline & & Entera & 18,01 & 15,62 & 55,84 & 12,80 \\
\hline & \multirow[t]{3}{*}{ Limpia } & Hoja & 17,80 & 24,85 & 38,30 & 18,37 \\
\hline & & Tallo & 15,36 & 9,77 & 71,18 & 8,59 \\
\hline & & Entera & 16,60 & 17,95 & 53,48 & 13,89 \\
\hline \multirow[t]{9}{*}{120} & \multirow[t]{3}{*}{ Testigo } & Hoja & 16,88 & 23,61 & 39,85 & 17,42 \\
\hline & & Tallo & 18,25 & 8,84 & 74,62 & 7,69 \\
\hline & & Entera & 17,61 & 15,53 & 57,78 & 12,41 \\
\hline & \multirow[t]{3}{*}{ Semilimpia } & Hoja & 18,01 & 22,30 & 34,71 & 18,44 \\
\hline & & Tallo & 16,54 & 8,06 & 71,33 & 8,27 \\
\hline & & Entera & 17,29 & 14,72 & 52,20 & 13,57 \\
\hline & \multirow[t]{3}{*}{ Limpia } & Hoja & 18,51 & 24,25 & 35,44 & 18,30 \\
\hline & & Tallo & 15,87 & 6,63 & 70,13 & 8,48 \\
\hline & & Entera & 17,16 & 17,03 & 52,11 & 13,53 \\
\hline
\end{tabular}

\section{CONCLUSIONES Y RECOMENDACIONES}

La poda semilimpia provocó una pérdida del 10\% en la producción de materia seca de hoja por hectárea y un $13 \%$ en el rendimiento de tallo con respecto al tratamiento testigo. La cosecha conjunta de hojas y tallos decreció un $11 \%$. La poda limpia provocó una disminución de $32 \%$ en el rendimiento de materia seca de hoja por hectárea y $36 \%$ en la producción de tallo con relación al tratamiento testigo. La producción global por área bajó 33\%. 
Se recomienda efectuar la poda semilimpia para eliminar la corona de rebrote y permitir el paso entre hileras o surcos en cualquiera de las densidades de siembra estudiadas. La poda limpia se recomienda realizar cuando se tiene interés en darle una conformación particular a cada planta individual.

\section{LITERATURA CONSULTADA}

A.O.A.C. (Association of Official Analysis Chemistry). 1980. Methods of analysis. $13^{\text {th }}$ ed. Washington D.C. EUA, AOAC. p. 168.

BENAVIDES, J.; BOREL, R.; ESNAOLA, M.A. 1986. Evaluación de la producción de forraje del árbol de morera (Morus sp.) sometido a diferentes frecuencias y altura es de corte. Inst. Centro Agronómico Tropical de Investigación y Enseñanza. Resumen de las investigaciones realizadas con rumiantes menores, en el Proyecto de Sistemas de Producción Animal. Serie Técnica. Informe Técnico 67: 74-76.

BENAVIDES, J.; LACHAUX, M.; FUENTES, M. 1994 Efecto de la aplicación de estiércol de cabra en el suelo sobre la calidad y producción de biomasa de morera (Morus sp.). Árboles y Arbustos Forrajeros en América Central. Serie Técnica. CATIE. Informe Técnico 236 (2): 495-514.

BLANCO, R. 1992. Distancia de siembra y altura de corte en la producción y calidad del forraje de Morera ( Morus sp.) en el parcelamiento Cuyunta, Escuintla Guatemala. Guatemala. Universidad de San Carlos. 15 p.

BOSCHINI, C.; DORMOND, H.; CASTRO, C. 1998. Producción de biomasa de morera (Morus alba) en la Meseta Central de Costa Rica. Agronomía Mesoamericana 9 (2): 31-40.

BOSCHINI, C.; DORMOND, H.; CASTRO, C. 2000. Composición química de la morera (Morus alba), para uso en la alimentación animal: Densidades y frecuencias de poda. Agronomía Mesoamericana 11 (1): 41-49.

DATTA, R. 2000. Mulberry cultivation and utilization in India. FAO Electronic Conference "Mulberry for anima production". May 1 to July 31, 2000. http://www.fao.org/WAICENT/FAOINFO/AGRICULT/AGA/AGAP/FRM/MULBERRY/ Papers/HTML/Datta.htm.

GOERING, H. K.; VAN SOEST, P.J. . 1970. Forage fiber analysis (apparatus, reagents, procedures and some applications). Agricultural Handbook No. 376. Washington, DC. ARS-USDA. 76 p.

GONZÁLEZ, F. 1951. El gusano de seda y la morera. 4a ed. Madrid, España. Ministerio de Agricultura. 272 p.

IFA (INTERNATIONAL FERTILIZER INDUSTRY ASSOCIATION). 1992. IFA World Fertilizer Use Manual. Mulberry Chart. BASF. Aktiengesells-Chaft. Agricultural Research Station. Germany. p. 595-601.
LIN, J.T.; YU, S.J. ; HSIEH, F.K. 1994. Effects of plant spacing on the yield and chemical compositions of mulberry leaves. Journal of the Agricultural Association of China 167:43-49.

RODRÍGUEZ, C.; ARIAS, R.; QUIÑONES J. 1994. Efecto de la frecuencia de poda y el nivel de fertilización nitrogenada, sobre el rendimiento y calidad de la biomasa de morera (Morus sp) en el trópico seco de Guatemala. Árboles y arbustos forrajeros en América Central. Serie Técnica. CATIE. Informe Técnico 236 (2): 515528 .

SÁNCHEZ, M.D. 2000. World distribution and utilization of mulberry, potential for animal feeding. FAO Electronic Conference "Mulberry for animal production". May 1 to July 31, 2000. http://www.fao.org/WAICENT/FAOINFO/AGRICULT/AGA/AGAP/FRM/MULBERRY/ Papers/HTML/Intro.htm.

SAS. 1985. Statistical Analysis System. SAS User's Guide: Statistics. $5^{\text {th }}$ ed. Cary, N.C., USA. SAS Institute Inc. $373 \mathrm{p}$.

SUZUKI, T.; KOHNO, K. 1987. Effects of pruning on the branching habit on Morus alba L. and the abscission of the apices of the short shoots. The New Phytologist 106: 753-758.

SUZUKI, T.; KITANO, M.,; KOHNO, K. 1988. Lateral bud outgrowth on decapitated shoots of low-pruned mulberry (Morus alba L.). Tree Physiology 4:53-60 p.

TAIZ, L.; ZEIGER, E. 1991. Plant physiology. The Benjamin/Cummings Publishing Company, Inc. California, USA. $645 \mathrm{p}$.

TING-ZING，Z.; YUN-FAN， T.; GUANG-XIEN， H.; HUAIZHONG, F.; BEN, MA. 1988. Mulberry cultivation. FAO Agricultural Services Bulletin 73/1. Food and Agricultural Organization of the United Nations. Rome. p. 127.

VASQUEZ, A. 1982. Estudio detallado de los suelos de la Estación Experimental de Ganado Lechero El Alto. Escuela de Fitotecnia, Facultad de Agronomía, Universidad de Costa Rica. p. 36.

YAMASHITA, T. 1984. Effect of top pruning on stem carbohydrates reserves and foliar $14 \mathrm{CO}_{2}$ (carbon dioxide isotope) assimilation in mulberry trees during autumn. Plant and Cell Physiology 25:677-681.

YAMASHITA, T.; FUJINO, A. 1986. Effects of pruning of young and old shoots on ribose bisphosphate carboxilase and other constutuents in leaves of the mulberry tree (Morus alba L.). Journal of Experimental Botany 37: 1836-1841.

YONGKANG HUO. 2000. Mulberry cultivation and utilization in China. FAO Electronic Conference "Mulberry for animal production". May 1 to July 31, 2000. http://www.fao.org/WAICENT/FAOINFO/AGRICULT / A G A / A G A P / F R M / M U L B E R R Y / P a pers/HTML/YONGKANG.htm. 